\title{
A NOTE ON THE DQ ANALYSIS OF ANISOTROPIC PLATES
}

WEN CHEN", WEIXING HE**, AND TINGXIU ZHONG*

"Affiliation: Department of Mechanical Engineering, Shanghai Jiao Tong University, Shanghai 200030, P. R. China

${ }^{* *}$ Affiliation: Department of Electrical Engineering, Jiangsu University of Science \& Technology, Zhenjiang, Jiangsu 212013, P. R. China.

Corresponding author: Wen Chen (PhD), Mail address: P. O. Box 9601BB, Shanghai Jiao Tong University, Shanghai 200030, P. R. China. Fax: 0086-021-62829425, Tel: 0086-02162812679, E-mail: ctwang@sjtu.edu.cn

The total number of pages is 6 . 


\section{INTRODUCTION}

Recently, Bert, Wang and Striz [1, 2] applied the differential quadrature (DQ) and harmonic differential quadrature (HDQ) methods to analyze static and dynamic behaviors of anisotropic plates. Their studies showed that the methods were conceptually simple and computationally efficient in comparison to other numerical techniques. Based on some recent work by the present author $[3,4]$, the purpose of this note is to further simplify the formulation effort and improve computing efficiency in applying the $D Q$ and $H D Q$ methods for these cases.

\section{APPROXIMATE FORMULAS IN MATRIX FORM AND REDUCTION COMPUTATIONS}

The details about the $\mathrm{DQ}$ and $\mathrm{HDQ}$ methods see reference [1]. The only difference between $\mathrm{DQ}$ and HDQ methods is to choose different basis functions, namely, the former is based on the polynomials and the latter based on harmonic functions.

\subsection{Approximate formulas in matrix form}

Approximate formulas for partial derivatives of function $w(x, y)$ in two-dimensional domain are given in matrix form by [3]

$$
\begin{aligned}
& \frac{\partial^{4} \hat{W}}{\partial x^{4}}=\bar{D}_{x} \hat{W}, \quad \frac{\partial^{4} \hat{W}}{\partial x^{3} \partial y}=\bar{C}_{x} \hat{W} \bar{A}_{y}^{T}, \quad \frac{\partial^{4} \hat{W}}{\partial x^{2} \partial y^{2}}=\bar{B}_{x} \hat{W} \bar{B}_{y}^{T}, \quad \frac{\partial^{4} \hat{W}}{\partial x \partial y^{3}}=\bar{A}_{x} \hat{W} \bar{C}_{y}^{T}, \\
& \frac{\partial^{4} \hat{W}}{\partial y^{4}}=\hat{W} \bar{D}_{y}^{T}, \quad \frac{\partial^{2} \hat{W}}{\partial x^{2}}=\bar{B}_{x} \hat{W}, \quad \frac{\partial^{2} \hat{W}}{\partial y^{2}}=\hat{W} \bar{B}_{y}^{T}
\end{aligned}
$$

where the unknown $\hat{W}$ is a $\mathrm{n} \times \mathrm{m}$ rectangular matrix rather than a vector as in references $[1,2], \mathrm{n}$ and $\mathrm{m}$ is the number of inner grid points along $\mathrm{x}$ - and $\mathrm{y}$ - directions, respectively. $\bar{A}, \bar{B}, \bar{C}$ and $\bar{D}$ with subscripts $\mathrm{x}$ and $\mathrm{y}$ here stand for the $\mathrm{DQ}$ weighting coefficient matrices, modified by the respective boundary conditions using Wang and Bert's new approach [5], for the 1st, 2nd, 3rd and 4th order partial derivatives, respectively. The superscript $T$ means the transpose of the matrices. It is noted that present DQ approximate formulas in matrix form can be easily extended to three-dimensional problems. The desired $\hat{W}$ in rectangular matrix form can be converted into the conventional vector form by the following Lemma 1. 
Lemma 1. If $A \in C^{p \times m}, B \in C^{n \times q}$ and the unknown $X \in C^{m \times n}$, then

$\operatorname{vec}(A X B)=\left(A \otimes B^{T}\right) \operatorname{vec}(X)$

where $\operatorname{vec}()$ is the vector-function of a rectangular matrix formed by stacking the rows of matrix into one long vector, $\otimes$ denotes the Kronecker product of matrices. To simplify the presentation, we define $\operatorname{vec}(A X B)=A X \vec{B}$ and $\operatorname{vec}(X)=\vec{X}$.

Corollary:

$1 A \vec{X}=\left(A \otimes I_{n}\right) \vec{X}$

$2 X \vec{B}=\left(I_{m} \otimes B^{T}\right) \vec{X}$

$3 A \vec{X}+X \vec{B}=\left(A \otimes I_{n}+I_{m} \otimes B^{T}\right) \vec{X}$

where $I_{n}$ and $I_{m}$ are the unit matrix.

\subsection{Centrosymmetric matrices and computing reduction}

The weighting coefficient matrices in the $\mathrm{DQ}$ and $\mathrm{HDQ}$ methods were proven to be either centrosymmetric matrix for the derivatives of even order or skew centrosymmetric matrix for the derivatives of odd order if a grid spacing is symmetric $[3,4]$. This is often seen in many situations such as equally spaced grid points or zeros of the Chebyshev and the Legendre polynomials. In the following we establish the notations on the centrosymmetric and skew centrosymmetric matrices first $[3,4,6]$.

Definition 1. Let $V_{N \times N}$ and $N V_{N \times N}$ denote the set of $N \times N$ real centrosymmetric and skew centrosymmetric matrices, respectively, then $X=\left[x_{i j}\right] \in V_{N \times N}$ if and only if $x_{N+1-i, N+1-j}=x_{i j}$; and $X=\left[x_{i j}\right] \in N V_{N \times N}$ if and only if $x_{N+1-i, N+1-j}=-x_{i j}$.

Lemma 2. If $Q_{1}, Q_{2} \in V_{N \times N} ; Q_{3}, Q_{4} \in N V_{N \times N}$, then $Q_{1} Q_{2} \in V_{N \times N}, Q_{1}+Q_{2} \in V_{N \times N}, Q_{3} Q_{4} \in V_{N \times N}$ 
The following lemma 3 is presented on the Kronecker product of the centrosymmetric and skew centrosymmetric matrices. The proofs are straightforward and thus omitted for the sake of brevity.

Lemma 3. If $A_{1}, A_{2} \in V_{N \times N}$ and $B_{1}, B_{2} \in N V_{N \times N}$, then $A_{1} \otimes A_{2} \in V_{N \times N}, B_{1} \otimes B_{2} \in V_{N \times N}, A_{1} \otimes B_{1} \in N V_{N \times N}$.

The centrosymmetric and skew centrosymmetric matrices can be factorized into two smaller sub matrices in the calculation of their determinant, inverse and eigenmodes. Therefore, the respective computing effort and storage requirements can be reduced by 75 percent and 50 percent, respectively. The details on centrosymmetric and skew centrosymmetric matrices can be found in references $[3,4,6]$.

\section{ON ANISOTROPIC PLATES}

The equation governing the behaviors of mid-plane symmetric laminated plates is given by [1]

$$
\begin{aligned}
\bar{D}_{11} w_{, x x x x}+4 \bar{D}_{16} w_{, x x x y}+ & 2\left(\overline{D_{12}}+\overline{D_{66}}\right) w_{, x x y y}+4{\overline{D_{26}}}_{2 w_{, x y y y}}+\bar{D}_{22} w_{, y y y y} \\
& =q+\rho h \omega^{2} w-N_{x} w_{, x x}-N_{y} w_{, y y}-2 N_{x y} W_{, x y}
\end{aligned},
$$

where $\bar{D}_{i j}$ are the plate stiffness, $\mathrm{h}$ is the total plate thickness, $\rho$ is the density, $\mathrm{w}$ is the model deflections, $q$ is the pressure only for deflection analysis, $\omega$ is the natural frequency only for free vibration analysis, $\mathrm{N}_{\mathrm{x}}$ and $\mathrm{N}_{\mathrm{y}}$ are uniform compression in-plane loads in the $\mathrm{x}$ - and $\mathrm{y}$ - directions for buckling analysis.

In terms of the present $\mathrm{DQ}$ and $\mathrm{HDQ}$ approximate formulas (1) with $\bar{N}=N_{x}=N_{y}$ and $N_{x y}=0$, we have

$$
\begin{aligned}
\bar{D}_{11} \bar{D}_{x} \hat{w}+4 \bar{D}_{16} \beta \bar{C}_{x} \hat{w} \bar{A}_{y}^{T} & +2 \beta^{2}\left(\bar{D}_{12}+\bar{D}_{66}\right) \bar{B}_{x} \hat{w} \bar{B}_{y}^{T}+4 \bar{D}_{26} \beta^{3} \bar{A}_{x} \hat{w} \bar{C}_{y}^{T} \\
& +\bar{D}_{22} \beta^{4} \hat{w} \bar{D}_{y}^{T}=q a^{4}+\bar{\varpi}^{2} \hat{w}-\bar{N} a^{2}\left(\bar{B}_{x} \hat{w}+\hat{w} \bar{B}_{y}^{T}\right)^{\prime}
\end{aligned}
$$


where $\beta=a / b$ denotes the aspect ratio, $\Phi^{2}=\rho h a^{4} \omega^{2}$. The relative boundary conditions have been taken into account in the formulation of weighting coefficient matrices, no additional equations are required.

Applying Lemma 1 and relative corollaries yields

$$
\begin{array}{r}
{\left[\bar{D}_{11}\left(\bar{D}_{x} \otimes I_{y}\right)+4 \bar{D}_{16} \beta\left(\bar{C}_{x} \otimes \bar{A}_{y}\right)+2 \beta^{2}\left(\bar{D}_{12}+\bar{D}_{66}\right)\left(\bar{B}_{x} \otimes \bar{B}_{y}\right)+4 \bar{D}_{26} \beta^{3}\left(\bar{A}_{x} \otimes \bar{C}_{y}\right)\right.} \\
\left.+\bar{D}_{22} \beta^{4}\left(I_{x} \otimes \bar{D}_{y}\right)\right] \vec{W}=q a^{4}+\bar{D}^{2} \vec{W}-\bar{N} a^{2}\left(\bar{B}_{x} \otimes I_{y}+I_{x} \otimes \bar{B}_{y}\right) \vec{W}
\end{array} .
$$

The above formulation equation is equivalent to equation (13) in references [1, 2]. The present procedures obviously simplify formulation effort and are much easier for programming, and the resulting formulation has an explicit matrix form. It can be concluded that the approximate formulas in matrix form in the $D Q$ and $H D Q$ methods are much simpler, more compact and convenient to formulate partial differential operators in two-dimensional domain than the conventional ones in polynomial form presented by Civan and Sliepcevich [7].

For problems with symmetric boundary conditions such as the simply supported or clamped anisotropic plates as discussed in references [1, 2], it is straightforward that $\bar{A}_{\mathrm{x}}, \bar{A}_{\mathrm{y}}, \bar{C}_{\mathrm{x}}$ and $\bar{C}_{\mathrm{y}}$ are skew centrosymmetric matrix and $\bar{B}_{\mathrm{x}}, \bar{B}_{\mathrm{y}}, \bar{D}_{\mathrm{x}}$ and $\bar{D}_{\mathrm{y}}$ are centrosymmetric matrix when the uniform grid points or the zeros of the Chebyshev polynomials are used. According to Lemmas 2 and 3, the resulting coefficient matrix in the formulation equation (6) is a centrosymmetric matrix. Therefore, the reduction algorithm based on the factorization properties of centrosymmetric matrix $[3,4,6]$ is applicable for the cases, namely, the computing effort and storage requirements are reduced to 75 percent and 50 percent as much as that in references [1, 2].

Bert et al. [1, 2] pointed out that the $D Q$ and $H D Q$ methods were very competitive technique to analyze static and dynamic behaviors of the anisotropic plates. The present work makes the methods more computationally efficient and easier to be used for these problems. 


\section{REFERENCES}

1) C. W. Bert, X. Wang and A. G. Striz 1993 International Journal of Solids and Structures 30, 1737-1744. Differential quadrature for static and free vibration analysis of anisotropic plates.

2) C. W. Bert, X. Wang and A. G. Striz 1994 Journal of Sound and Vibration 170, 140-144. Convergence of the $\mathrm{DQ}$ method in the analysis of anisotropic plates.

3) Wen Chen, Yongxi Yu and Xinwei Wang 1996 Numerical Methods for Partial Differential Equations 12, 565-577. Reducing the computational effort of the differential quadrature method.

4) Wen Chen, Xinwei Wang and Tingxiu Zhong 1996 Communications in Numerical Methods in Engineering 12, 455-460. The structure of weighting coefficient matrices of harmonic differential quadrature and its applications.

5) X. Wang and C. W. Bert 1993 Journal of Vibration and Sound 162, 566-572. A new approach in applying differential quadrature to static and free vibrational analysis of beams and plates.

6) L. Datta and S. D. Morgera 1989 Circuits Systems Signal Process 8, 71-96. On the reducibility of centrosymmetric matrices applications in engineering problems.

7) F. Civan and C. M. Sliepcevich 1984 Journal of Mathematical Analysis and Applications 101, 423-443. Differential quadrature for multi-dimensional problems. 